\section{Assessment of Future Heat Stress Risk in European Regions: Towards a better Integration of Socio- economic Scenarios}

Gl_Forum 2017, Issue 1

Page: 341 - 351

Short Paper

Corresponding Author:

guillaume.rohat@unige.ch

DOI: 10.1553/giscience2017_01_s341

\author{
Guillaume Rohat1, 2, Johannes Flacke² and Hy Dao 1, 3 \\ 'Institute for Environmental Sciences, University of Geneva, Switzerland \\ 2Faculty of Geo-Information Science and Earth Observation, University of Twente, The \\ Netherlands \\ 3United Nations Environment Programme DEWA/GRID-Geneva, Switzerland
}

\begin{abstract}
The overwhelming majority of integrated assessments of future climate risks are made using climate scenarios and projections superimposed on current socio-economic conditions only; hence they fail to account for the influence of socio-economic changes. Following the recent IPCC-related new scenarios framework for climate change research, a few assessments of climate risks have attempted to integrate socio-economic changes through the combination of climate and socio-economic scenarios. However, a number of shortcomings remain, such as the lack of consideration of vulnerability, the low spatial resolution, and the lack of contextual focus. In this paper, we seek to examine these shortcomings through an exploratory assessment of future heat stress risk in 271 European regions up to 2030, based on the combination of several climate and socio-economic scenarios. We also discuss the main barriers faced - such as the limited number of socioeconomic projections carried out to date and the diversity of existing socioeconomic scenarios - and provide a reflection on promising approaches to foster the use of socio-economic projections and scenarios within integrated assessments of future climate risks.
\end{abstract}

\title{
Keywords:
}

heat stress risk, scenarios, vulnerability, ilntegrated assessment, Europe

\section{Introduction}

Human-induced climate change is undoubtedly one of the most significant threats for human beings and societies. Understanding how changes in climatic conditions are jeopardizing public health and well-being has been at the forefront of climate change research in recent decades. Most of the assessments of climate risks conducted nowadays are integrated, meaning that they recognize the influence of both climatic and socio-economic conditions on climate risks. However, more often than not, they only consider temporal changes in climatic conditions and do not integrate changes in socio-economic conditions; 
hence they fail to account for the influence that socio-economic development has on future risks and vulnerabilities (Garschagen and Kraas, 2010; Rohat et al., 2016).

To foster the use of socio-economic projections in assessments of climate risks, the climate change research community has been recently engaged in the development of a new scenarios framework, comprising climate scenarios - namely Representative Concentration Pathways (RCPs) - and socio-economic scenarios - namely Shared Socio-economic Pathways (SSPs) (Moss et al., 2010). These climate and socio-economic scenarios are purposely designed to be combined in a scenarios matrix (Kriegler et al., 2014) to explore future climate risks, i.e. to create scenarios of risks and vulnerabilities (Birkmann et al., 2013).

The rapidly growing number of assessments of climate risks making use of this approach often present a range of shortcomings, such as (i) little consideration of vulnerability: most of the studies use only projections of exposure (i.e. population density or GDP for economic exposure) and do not account for vulnerability; (ii) the low spatial resolution of the assessments, which are mostly based on national projections; (iii) the lack of contextual focus, meaning that numerous regional assessments of climate risks simply apply global socio-economic scenarios (such as the SSPs) without accounting for the local context.

We aimed to address these shortcomings through an exploratory integrated assessment of future heat stress risk in Europe, attempting to: (i) integrate projections of vulnerability variables; (ii) assess future heat stress risk at a sub-national spatial scale; (iii) account for the local context through the use of European socio-economic scenarios. In this paper, we introduce the methods applied to compute future heat stress risk, discuss the results, and provide some reflections regarding the barriers faced and the promising approaches which might overcome them.

\section{Methods}

Based on the latest definition of risk and vulnerability (IPCC, 2012), we defined heat stress risk as the combination of exposure, vulnerability and heat stress. While heat stress is defined by climatic variables, human exposure and vulnerability are both driven by socio-economic variables only. Computations of relevant indicators for these three pillars of risk were made as follows, up to 2030, for each of the 271 administrative European regions (also known as NUTS2 regions, i.e. territorial units for statistics in Europe).

\section{Heat stress index and climate scenarios}

A great number of heat stress indices have been described in the literature (Bao et al., 2015). In this study, as a heat stress index we used the changes in average daily maximum temperatures during the summer months (June, July and August), from the reference period (2000-2010) to the projected period (2025-2035). Such an indicator of heat stress has already been applied across Europe (Greiving, 2011) and allows the identification of regions that face great changes in high summer temperatures regardless of their initial temperature (Dong et al., 2015). 
Computations were made under two contrasting IPCC climate scenarios, namely RCP2.6 and RCP8.5. Under the latter scenario, emissions rates of greenhouse gases show a continuous growth, while they decrease slightly by 2030 under RCP2.6. Logically, a mean temperature increase is expected to be higher under RCP8.5.

Bias-corrected ensembles of Regional Climate Model simulations for these two RCPs were retrieved from the EURO-CORDEX initiative (Jacob et al., 2014) and served as inputs for computing the heat stress index.

\section{Exposure, vulnerability and socio-economic scenarios}

Unlike climatic variables, quantitative projections of socio-economic variables are rather scarce. Numerous European forward-looking studies exist (EEA, 2011), but not all of them are associated with quantitative projections at NUTS2 scale, and they are based on different sets of socio-economic scenarios (which renders difficult any co-use of their quantitative outputs). In this study, we made use of (i) the global SSPs, for which a few key socioeconomic variables have been quantified at the national level (O'Neill et al., 2015), and (ii) the ET2050-Territorial Scenarios and Visions for Europe (Ulied et al., 2014), in which four socio-economic scenarios (and different variants) have been developed and quantified.

Based on a matching by archetypes (Hunt et al., 2012), we successfully linked the narratives of two distinct ET2050 scenarios with SSP2 and SSP5. Although frequently applied to compare and group scenarios (van Vuuren and Carter, 2013), such qualitative matching is somewhat approximate. Thus, systematic methods to match scenarios' narratives in a more precise and structured manner are needed in the future. SSP2 and SSP5 depict very different futures: SSP2 stands for a business-as-usual scenario in which there is medium economic and population growth, a European landscape development based on existing patterns, low/medium social cohesion and equity, and medium challenges for both climate adaptation and mitigation; SSP5 presents high economic growth based on fossil-fuel consumption, high population growth, a European landscape structure clustered around urban development, high social cohesion and equity, as well as low challenges for climate adaptation but high challenges for climate mitigation.

There is no consensus regarding either the methods to identify and select indicators of exposure and vulnerability (Yoon, 2012), or the methods to combine them, although best practices are starting to emerge (Reckien, in review). In this study, we applied a deductive approach based on a theoretical understanding from the literature (Bao et al., 2015) that led us to select population density as an indicator of population exposure, and four other socioeconomic variables as indicators of vulnerability, namely the gross domestic product (GDP) per capita, the old-dependency-ratio (ODR), the proportion of built-up areas, and the proportion of 25- to 64-year-olds with higher education. While projections for the last of these variables were downscaled from SSPs' national values (assuming that sub-national educational trends follow the national trend (Xing et al., 2015)), projections for the other four socio-economic variables were available at NUTS2 scale from the ET2050 project.

To compute the socio-economic dimension of heat stress risk (i.e. the combination of exposure and vulnerability), we used an additive model without weighting. After a common 
min-max normalization, socio-economic determinants of heat stress risk were combined as follows:

Combined exposure-vulnerability $=$

$1 / 5 *[($ Pop density $)+(O D R)+(\%$ Built-up areas $)+(1-G D P)+(1-\%$ Higher education $)]$

The socio-economic dimension of heat stress risk was computed for the year 2015 according to current socio-economic conditions and for the year 2030 according to SSP2 and SSP5, for each of the 271 NUTS2 regions.

\section{Heat stress risk and scenarios combinations}

We defined heat stress risk as being the multiplicative combination of the normalized socioeconomic and climatic dimensions. Some combinations of climate and socio-economic scenarios are more likely than others, e.g. the socio-economic development depicted in SSP5 is rather unlikely to lead to the low emissions rates expected under scenario RCP2.6. Nevertheless, future heat stress risk was computed for the four possible combinations of scenarios as well as for the two combinations of climate scenarios with current socioeconomic conditions. Resulting risks values were normalized (also using min-max linear transformation) and classified into seven classes from low to high.

\section{Results}

As expected, the overall European heat stress index in 2030 (Figure 1) is greater under RCP8.5 than under RCP2.6. Nevertheless, heat stress is not homogeneously distributed across the European regions. Those located in the Iberian Peninsula, Finland and the Alps will face the most substantial changes in summer maximum temperatures under the two RCPs. 


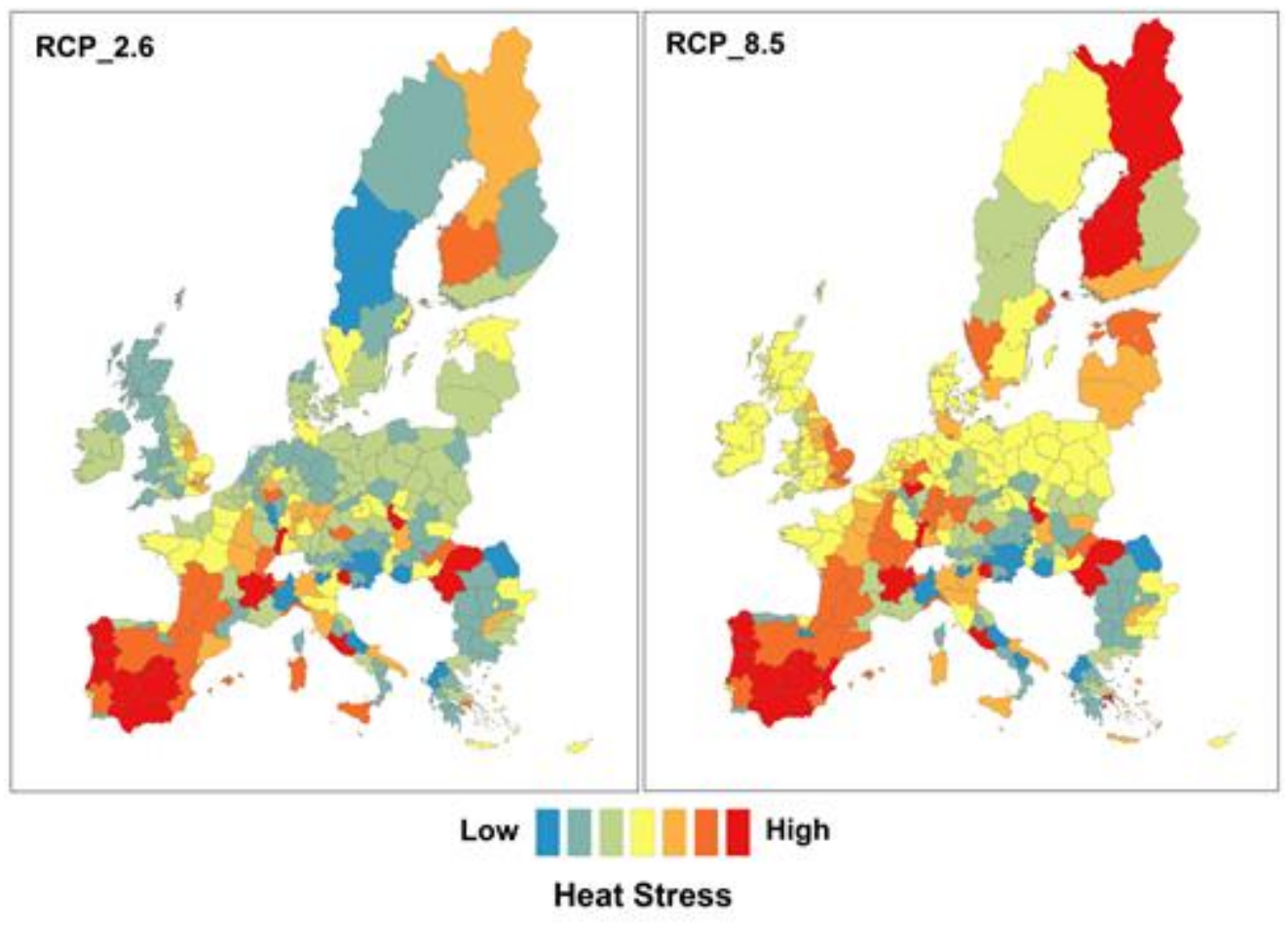

Figure 1: Spatial distribution of the normalized heat stress index, ranging from low (blue) to high (red), for the 271 NUTS2 European regions under RCP2.6 and RCP8.5 emissions scenarios, in 2030.

Computations of the combined exposure-vulnerability (Figure 2) show that (i) the two SSPs lead to significantly different patterns, and (ii) future combined exposure-vulnerability will be greater than in 2015 (labelled as current), for both SSPs.

Under the two SSPs, regions located in Portugal, Southern Italy, Romania and Central Europe (including various German regions) present the highest combined exposurevulnerability to heat stress. However, it is worth mentioning that the underlying drivers differ. For example, German regions' high combined exposure-vulnerability is due to a high population density, an ageing society, and a high degree of soil sealing, while the high combined exposure-vulnerability of Portuguese and Romanian regions is due mainly to low GDP per capita and low education levels. 


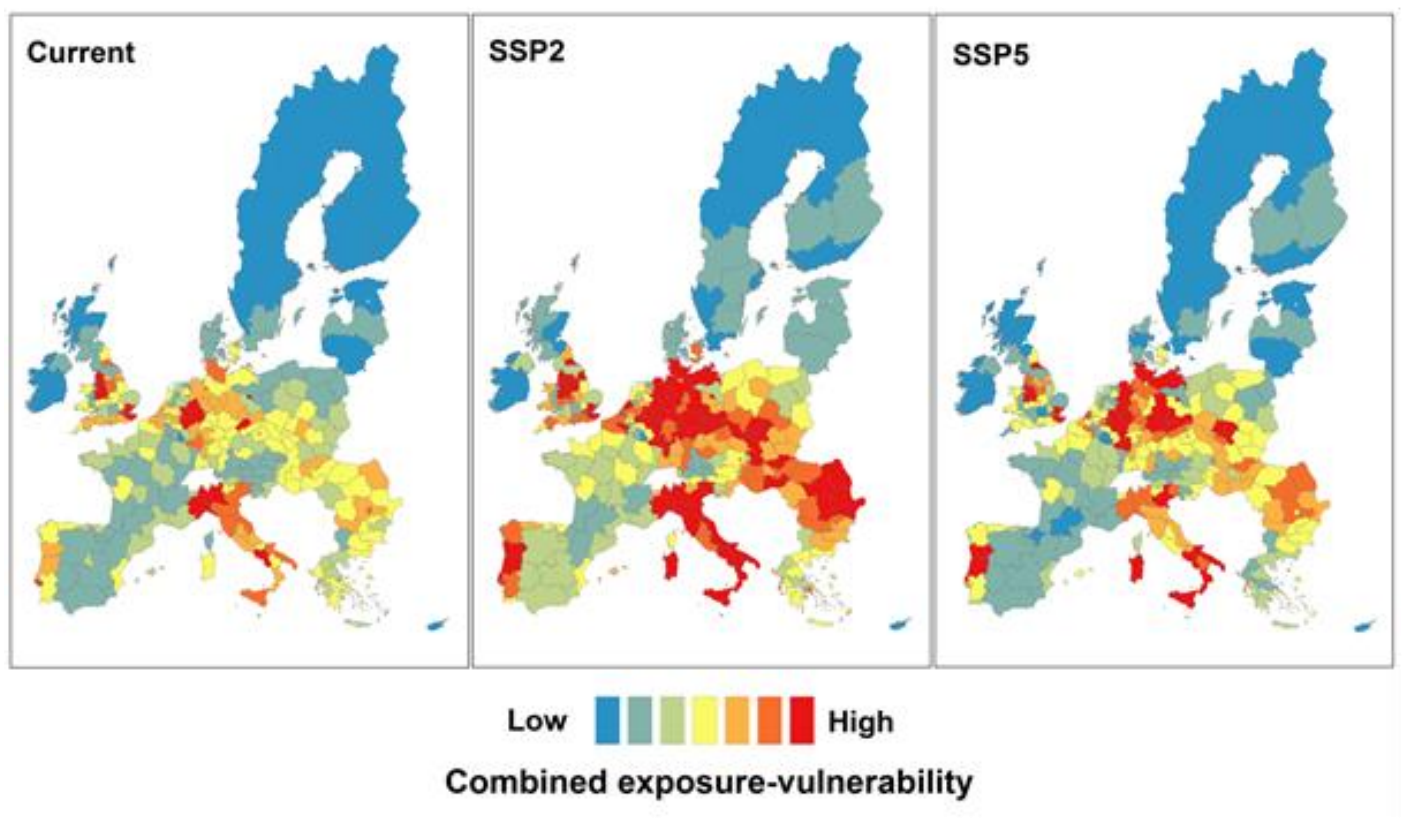

Figure 2: Spatial distribution of the normalized combined exposure-vulnerability, ranging from low (blue) to high (red), for the 271 NUTS2 European regions under current (2015) and future (2030) socioeconomic conditions according to socio-economic scenarios SSP2 and SSP5.

The final results of the integrated assessment (Figure 3) show that the combination of a high emissions scenario (RCP8.5) with a business-as-usual socio-economic scenario (SSP2) leads to the greatest heat stress risk in Europe, while the (very unlikely) combination of a low emissions scenario (RCP2.6) with a scenario of fossil fuel-based equitable economic growth (SSP5) leads to the lowest heat stress risk. However, large regional disparities can be seen. For instance, Nordic regions show very little heat stress risk, even under the RCP8.5*SSP2 combination, due to their very low combined exposure-vulnerability. In contrast, regions located in Portugal, Italy and Romania exhibit a high heat stress risk under all scenarios combinations. As regards regions of Central Europe, their levels of future heat stress risk differ significantly from one combination of scenarios to another, underlining the joint influence of climatic and socio-economic changes on future heat stress risk. 


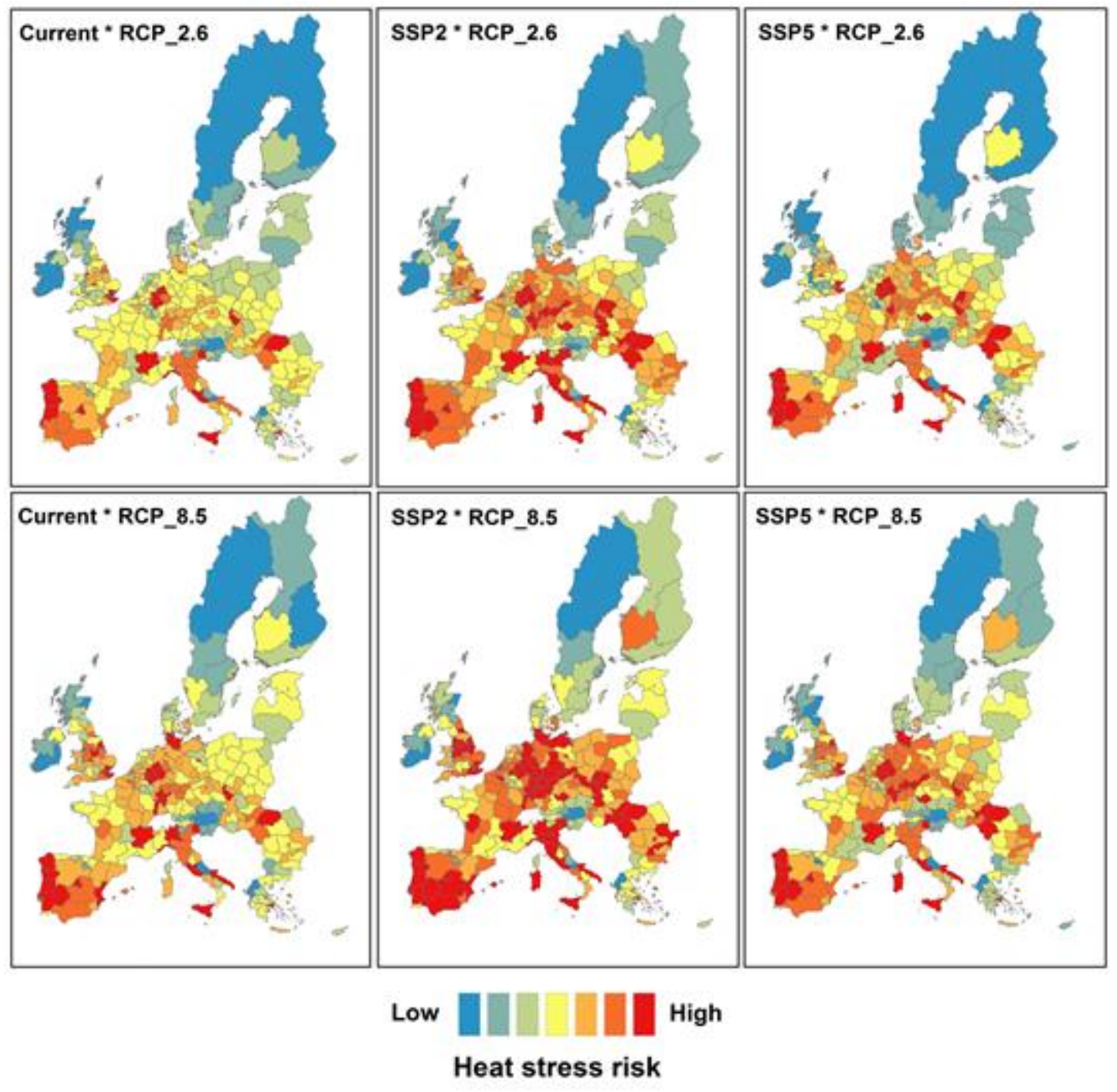

Figure 3: Spatial distribution of the normalized heat stress risk, ranging from low (blue) to high (red), for the 271 NUTS2 European regions under different combinations of climate and socio-economic scenarios (2030) and current conditions (2015).

\section{$4 \quad$ Discussion and Conclusion}

In our attempt to address the aforementioned shortcomings, two main barriers were encountered. 


\section{Lack of data for socio-economic projections}

This is the most obvious barrier explaining the lack of use of socio-economic projections within integrated assessments. Compared to climatic variables, there is indeed a considerable lack of quantitative projections of socio-economic variables, in terms of diversity (available projections are often limited to GDP and population growth), spatial scale (projections are often available at national scale only), and temporal scale (post-2050 projections are very scarce). However, we argue here that among the large number of forward-looking studies, quantitative and reliable projections of key socio-economic determinants of vulnerability (such as age, education and land use) at sub-national scale up to 2030 or 2050 are readily available, at least in developed countries. In addition, changes over time of socio-economic variables that matter when assessing climate risks are being increasingly studied and quantified by the climate change research community; hence the availability, reliability and diversity of socio-economic projections may increase rapidly in the next few years. Furthermore, a number of sectoral and integrated models exist and may constitute very helpful tools to produce reliable projections for essential socio-economic variables. Diverse methods of statistical downscaling could also be applied to obtain socio-economic projections at the desired unit of analysis, as already shown with previous IPCC SRES scenarios (Gaffin et al., 2004). Finally, although understanding fully the complexity of vulnerability requires the use of numerous socio-economic variables (Wannewitz et al., 2016), such complexity may be reduced through a condensed vulnerability index made of proxy variables that can be projected through their correlation with existing projections of common variables such as GDP and population growth (Kienberger et al., 2015).

\section{Variety of existing socio-economic scenarios}

In the frame of this exploratory study, we identified several dozen forward-looking studies that have produced quantitative projections for various socio-economic variables in Europe. These numerous projections (at various temporal and spatial scales) have the potential to constitute a very rich database that could enhance the integration of future socio-economic conditions into risk assessments. However, forward-looking studies that produce socioeconomic projections are often based on different sets of socio-economic scenarios. Such differences between the scenarios are a major hindrance to a co-use of these quantitative projections. In this paper, we argue that an effort must be made to link and match the different sets of socio-economic scenarios. We have successfully matched two ET2050 scenarios with two SSP scenarios, but this was achieved only through a qualitative matching of the narratives. More systemic scenario-matching methods - such as the linked-cross impact balance analysis (Schweizer \& Kurniawan, 2016) - have the potential to reliably match different sets of socio-economic scenarios. This would enable the co-use of their quantitative projections within assessments of climate risks. Finally, particular attention should be drawn to the consideration of the regional context, which can be done by matching existing socio-economic scenarios at different spatial scales (e.g. matching European scenarios with global scenarios such as the SSPs), and by extending the global SSPs into region-specific scenarios, such as the recently developed European SSPs (Kok et al., in review). 
Integrated assessments of future climate risks must consider changes in both climatic and socio-economic conditions. This can be achieved through the use of combinations of several climate and socio-economic scenarios, as suggested by the new scenarios framework for climate change research.

In this paper, we have applied such an approach to explore future heat stress risk in Europe under combinations of two climate and socio-economic scenarios. We have successfully (i) included projections of vulnerability drivers (namely age, education, GDP and land use), (ii) assessed future heat stress risk at the sub-national scale (NUTS2 regions), and (iii) accounted for the regional context through the matching of global and European scenarios.

The lack of available data for socio-economic projections and the large number of existing socio-economic scenarios have severely restricted our risk analysis. Nevertheless, we have argued that a certain number of methods and approaches may have the potential to overcome - at least partially - these main barriers. This will foster the use of socio-economic scenarios within assessments of climate risks, which is a crucial step for (i) better understanding how changes in climatic and socio-economic conditions will jointly affect future risk, and (ii) exploring which kind of socio-economic development will minimize climate risks the most, under different climatic conditions.

\section{Acknowledgements}

The authors wish to thank the two anonymous reviewers for their comments that helped improve the quality of this paper.

\section{References}

Bao, J., Li, X., \& Yu, C. (2015). The Construction and Validation of the Heat Vulnerability Index, a Review. International Journal of Environmental Research and Public Health, 12(7), pp.7220-7234. doi:10.3390/ijerph120707220

Birkmann, J., Cutter, S.L., Rothman, D.S., Welle, T., Garschagen, M., van Ruijven, B., O’Neill, B., Preston, B.L., Kienberger, S., Cardona, O.D., Siagian, T., Hidayati, D., Setiadi, N., Binder C.R., Hughes, B., \& Pulwarty, R. (2013). Scenarios for vulnerability: opportunities and constraints in the context of climate change and disaster risk. Climatic Change, 133(1), pp.53-68. doi:10.1007/s10584-013-0913-2

Dong, W., Liu, Z., Liao, H., Tang, Q., \& Li, X. (2015). New climate and socio-economic scenarios for assessing global human health challenges due to heat risk. Climatic Change, 130(4), pp.505-518. doi:10.1007/s10584-015-1372-8

EEA. (2011). Catalogue of scenario studies, ISSN 1725-2237, EEA Technical Report 1/2011, European Environment Agency, Copenhagen, 196 pp.

Gaffin, S.R., Rosenzweig, C., Xing, X., \& Yetman, G. (2004). Downscaling and geo-spatial gridding of socio-economic projections from the IPCC Special Report on Emissions Scenarios (SRES). Global Environmental Change, 14(2), pp.105-123. doi:10.1016/j.gloenvcha.2004.02.004

Garschagen, M. \& Kraas, F. (2010). Assessing future resilience to natural hazards - The challenges of capturing dynamic changes under conditions of transformations and climate change. International Disaster and Risk Conference 2010, Davos, Switzerland. 
Greiving, S. (2011). Climate change and territorial effects on regions and local economies. Scientific Report, ESPON Climate, 302pp. ISBN-978-2-919777-04-4

Hunt, D.V.L., Lombardi, D.R., Atkinson, S., Barber, A.R.G., Barnes, M., Bokyo C.T., Brown, J., Bryson, J., Butler, D., Caputo, S., Caserio, M., Coles, R., Cooper R.F.D., Farmani, R., Gaterell, M., Hale, J., Hales, C., Hewitt, C.N., Jankovic, L., Jefferson, I., Leach, J., MacKenzie, A.R., Memon, F.A., Sadler, J.P., Weingaertner, C., Whyatt, J.D., \& Rogers, C.D.F. (2012). Scenarios archetypes: Converging rather than diverging themes. Sustainability, 4(1), pp. 740-772. doi:10.3390/su4040740

IPCC (2012). Managing the risks of extreme events and disasters to advance climate change adaptation. Special report of the Intergovernmental Panel on Climate Change. Cambridge University Press, Cambridge, 594pp.

Jacob, D., Petersen, J., Eggert, B., Alias, A., Christensen, O.B., Bouwer, L.M., Braun, A., Colette, A., Deque, M., Georgievski, G., Georgopoulou, E., Gobiet, A., Menut, L., Nikulin, G., Haensler, A., \& Yiou, P. (2014). EURO-CORDEX: new high-resolution climate change projections for European impact research. Regional Environmental Change, 14, pp.563-578. doi:10.1007/s10113013-0499-2

Kienberger, S., Hutton, C. W., \& Amoako Johnson, F. (2015). Vulnerability assessment and scenarios. In: Sharma, N., \& Flügel, W.A. (eds) Applied geoinformatics for sustainable integrated land and water resources management (ILWRM) in the Brahmaputra River basin. Springer, New Delhi, pp.53-59. doi:10.1007/978-81-322-1967-5

Kok, K., Pedde, S., Gramberger, M., Harrison, P.A., \& Holman, I. (in review). New European socioeconomic scenarios for climate change research: Operationalising concepts to extend the Shared Socioeconomic Pathways. Regional Environmental Change, IMPRESSIONS Special Issue.

Kriegler, E., Edmonds, J., Hallegatte, S., Ebi, K.L., Kram, T., Riahi, K., Winkler, H., \& van Vuuren, D.P. (2014). A new scenario framework for climate change research: the concept of shared climate policy assumptions. Climatic Change, 122(1), pp.401-414. doi:10.1007/s10584-013-0971-5

Moss, R.H., Edmonds, J.A., Hibbard, K.A., Manning, M.R., Rose, S.K., van Vuurenm D.P., Carter, T.R, Emori, S., Kainuma, M., Kram, T., Meehl, G.A., Mitchell, J.F.B., Nakicenovic, N., Riahi, K., Smith S.J., Stouffer, R.J., Thomson, A.M., Weyant, J.P., \& Wilbanks, T.J. (2010). The next generation of scenarios for climate change research and assessment. Nature, 463(7282), pp.747756. doi: $10.1038 /$ nature 08823

O’Neill, B.C., Kriegler, E., Ebi, K.L., Kemp-Benedict, E., Riahi, K., Rothman, D.S., van Ruijven B.J., van Vuuren D.P., Birkmann, J., Kok, K., Levy, M., \& Solecki, W. (2015). The roads ahead: Narratives for shared socioeconomic pathways describing world futures in the 21 st century. Global Environmental Change. doi:10.1016/j.gloenvcha.2015.01.004

Reckien, D. (in review). Social vulnerability: a review and methodology check to move to social resilience. Regional Environmental Change

Rohat, G., Flacke, J., \& Dao, H. (2016). Application of the new scenario framework for climate change research: Future social vulnerability in large urban areas. Geophysical Research Abstracts, 18, EGU General Assembly 2016, Vienna. EGU2016-13323

Schweizer, V.J., \& Kurniawan, J.H. (2016). Systematically linking qualitative elements of scenarios across levels, scales, and sectors. Environmental Modelling \& Software. doi:10.1016/j.envsoft.2015.12.014

Ulied, A. (2014). ET2050 - Territorial scenarios and visions for Europe. Final Report, ESPON \& MCRIT, $121 \mathrm{pp}$.

van Vuuren, D.P. \& Carter, T.R. (2013). Climate and socioeconomic scenarios for climate change research and assessment: reconciling the new with the old. Climatic Change, 122(1), pp.415-429. doi:10.1007/s10584-013-0974-2.

Wannewitz, S., Hagenlocher, M., \& Garschagen, M. (2016). Development and validation of a subnational multi-hazard risk index for the Philippines. GI_Forum, 1, pp.133-140.

doi:10.1553/giscience2016_01_s133 


\section{Rohat et al}

Xing, R., Hanaoka, T., Kanamori, Y., Dai, H., \& Masui, T. (2015). An impact assessment of sustainable technologies for the Chinese urban residential sector at provincial level. Environmental Research Letters, 10, 065001. doi:10.1088/1748-9326/10/6/065001

Yoon, D.K. (2012). Assessment of social vulnerability to natural disasters: a comparative study.

Natural Hazards, 63(2), pp.823-843. doi:10.1007/s11069-012-0189-2 